\title{
Molecular Characterization and Patient Outcome of Melanoma Nodal Metastases and an Unknown Primary Site
}

\author{
Aleksandra Gos, BSc ${ }^{1}$, Monika Jurkowska, $\mathrm{PhD}^{4}$, Alexander van Akkooi, MD, $\mathrm{PhD}^{5}$, Caroline Robert, $\mathrm{MD}^{7}$, \\ Hanna Kosela-Paterczyk, $\mathrm{MD}^{2}$, Senada Koljenović, $\mathrm{MD}^{6}$, Nyam Kamsukom, $\mathrm{MD}^{7}$, Wanda Michej, $\mathrm{MD}^{3}$, \\ Arkadiusz Jeziorski, MD, $\mathbf{P h D}^{8}$, Piotr Pluta, $\mathbf{M D}^{8}$, Cornelis Verhoef, $\mathrm{MD}^{5}$, Janusz A. Siedlecki, MD, PhD ${ }^{1}$, \\ Alexander M. M. Eggermont, MD, $\mathbf{P h D}^{7}$, and Piotr Rutkowski, MD, $\mathbf{P h D}^{2}$ \\ ${ }^{1}$ Department of Molecular and Translational Oncology, Maria Sklodowska-Curie Memorial Cancer Center and Institute of \\ Oncology, Warsaw, Poland; ${ }^{2}$ Department of Soft Tissue/Bone Sarcoma and Melanoma, Maria Sklodowska-Curie \\ Memorial Cancer Center and Institute of Oncology, Warsaw, Poland; ${ }^{3}$ Department of Pathology, Maria Sklodowska-Curie \\ Memorial Cancer Center and Institute of Oncology, Warsaw, Poland; ${ }^{4}$ Institute of Rheumatology, Warsaw, Poland; \\ ${ }^{5}$ Department of Surgical Oncology, Erasmus MC Cancer Institute, Rotterdam, The Netherlands; ${ }^{6}$ Department of Pathology, \\ Erasmus University Medical Centre, Rotterdam, The Netherlands; ${ }^{7}$ Cancer Institute Gustave Roussy, Villejuif/Paris-Sud, \\ France; ${ }^{8}$ Medical University of Lodz, Lodz, Poland
}

\begin{abstract}
Background. Melanoma of unknown primary site (MUP) is not a completely understood entity with nodal metastases as the most common first clinical manifestation. The aim of this multicentric study was to assess frequency and type of oncogenic BRAF/NRAS/KIT mutations in MUP with clinically detected nodal metastases in relation to clinicopathologic features and outcome.

Materials and Methods. We analyzed series of 103 MUP patients (period: 1992-2010) after therapeutic lymphadenectomy (LND): 40 axillary, 47 groin, 16 cervical, none treated with BRAF inhibitors. We performed molecular characterization of BRAF/NRAS/KIT mutational status in nodal metastases using direct sequencing of respective coding sequences. Median follow-up time was 53 months. Results. BRAF mutations were detected in 55 cases (53\%) (51 V600E, $93 \%$; 4 others, $7 \%$ ), and mutually exclusive NRAS mutations were found in 14 cases (14\%)
\end{abstract}

\footnotetext{
The preliminary study was presented as an oral presentation during The 66th Annual Cancer Symposium of Society of Surgical Oncology, March 6-9, 2013, Washington, DC.
}

(C) The Author(s) 2014. This article is published with open access at Springerlink.com

First Received: 9 February 2014;

Published Online: 28 May 2014

P. Rutkowski, MD, PhD

e-mail: rutkowskip@coi.waw.pl
(7 p.Q61R, 4 p.Q61K, 2 p.Q61H, 1 p.Q13R). We have not detected any mutations in KIT. The 5-year overall survival (OS) was $34 \%$; median was 24 months. We have not found significant correlation between mutational status (BRAF/NRAS) and OS; however, for BRAF or NRAS mutated melanomas we observed significantly shorter disease-free survival (DFS) when compared with wild-type melanoma patients $(p=.04$; 5-year DFS, 18 vs 19 vs $31 \%$, respectively). The most important factor influencing OS was number of metastatic lymph nodes $>1(p=.03)$. Conclusions. Our large study on molecular characterization of MUP with nodal metastases showed that MUPs had molecular features similar to sporadic non-chronic-sundamaged melanomas. BRAF/NRAS mutational status had negative impact on DFS in this group of patients. These observations might have potential implication for molecular-targeted therapy in MUPs.

Metastatic involvement of lymph nodes is the most common clinical manifestation of melanoma of unknown primary site (MUP) and accounts for $\sim 3.2 \%$ of all melanoma cases, ranging from 1 to $15 \%$ of all melanomas with clinically detectable synchronous lymph nodes involvement. ${ }^{1-7}$ Currently, according to the American Joint Committee on Cancer (AJCC) guidelines, presentation of initial metastases in the lymph nodes should be presumed to be regional and staged accordingly (stage III instead of stage IV), if no other site of metastases is discovered during 
screening process. ${ }^{8}$ We hypothesize, the pathogenesis and molecular characteristics of MUP should be similar to melanomas with nodal metastases from known primary site.

It is now becoming clear that melanoma is not a homogeneous disease, but rather a group of neoplasms caused by different genetic changes and driven by different mechanisms. Patterns of known genetic changes differ significantly based on location of primary lesion and clinical melanoma subtype. Products of genes most commonly alternated in melanoma arising from the skin without chronic sun damage (NCSD, non-chronic sun damaged) are clustered in mitogen activated protein kinase (MAPK) pathway. ${ }^{9-11}$ In a majority of cases, the hyperactivation of MAPK pathway is caused by acquisition of oncogenic mutation in BRAF or NRAS genes. BRAF mutations are the most frequent changes in melanoma, and they comprise $40-70 \%$ of cases depending on melanoma type. ${ }^{12-14}$ More than 50 distinct mutations of BRAF gene were reported; $\sim 90 \%$ of them are due to a single nucleotide substitution T1799A at codon position 600 in exon 15 (p.V600E) leading to 500-fold increase in the protein activity. The second most frequent mutation is p.V600K, and it is less powerful as kinase activity increases. The important role of $B R A F$ alternations in melanoma development is proven; however, the mutation itself is not sufficient for malignant transformation, and BRAF mutations also occur with high frequency in benign nevi. ${ }^{15}$

The frequency of NRAS mutation in melanoma of cutaneous origin varies between 15 and $30 \% .{ }^{16}$ The majority of changes in this gene affect codon 61 (exon 2) as well as, to a lesser extent, codons 12 and 13 (exon 1). Similarly to $B R A F$, the NRAS mutations role in tumorigenesis is proven; however again, alone they are not sufficient to cause malignant transformation. BRAF and $N R A S$ mutations are mutually exclusive, which suggests functional redundancy in primary tumors. KIT alterations are rare and found mainly in melanomas on chronically sun-damaged skin, in acral-lentiginous or mucosal type. ${ }^{9}$

The prognostic role of alternations in BRAF and NRAS genes is not yet determined. Some reports imply association of BRAF/NRAS mutations and poorer prognosis in the metastatic setting. However, differences in disease-free survival (DFS) and overall survival (OS) according to BRAF/NRAS mutational status are not seen, when calculated from primary tumor diagnosis. ${ }^{11,17,18}$ Also data on survival in stage III melanoma patients according to NRAS mutations are not unanimous. ${ }^{18,19}$ Mutations in both genes are validated targets for molecular-targeted therapies in melanoma (BRAF inhibitors vemurafenib and dabrafenib for $B R A F$ mutants and MEK inhibitor trametinib for genotype containing any of the 2 genes altered). ${ }^{20-22}$ However, the molecular background of MUP, its linkage to clinical data, and differences from melanoma of known primary site are not fully understood, although they have been explored in recent series. ${ }^{23,24}$

In the current study we analyzed frequency and type of oncogenic mutations in known oncogenes $(B R A F, N R A S$, and $K I T$ ) involved in melanoma development in large contemporary series of MUP patients with long follow-up, and we correlated these outputs with disease clinical features and outcome. It may provide insight into molecular pathogenesis and characterization of this melanoma subtype.

\section{MATERIALS AND METHODS}

\section{Patients Characteristics}

Patients were considered eligible for the study if they had diagnosed clinical (palpable) nodal metastases of MUP, available tumor tissue, and underwent radical lymph node dissection (LND) at 1 of the centers participating in the study [Cancer Centre and Institute of Oncology, Warsaw, Poland (CCIO); Cancer Institute Gustave Roussy, Villejuif, France (IGR); Erasmus MC Cancer Institute, Rotterdam, Netherlands (Erasmus MC), and Medical University of Lodz, Poland (MU)].

The group of patients with MUP and lymph node metastases was defined as: metastases to the lymph nodes as first site of metastases, confirmed clinically, cytologically/histologically, and immunohistochemically; the absence of previous cutaneous tumors or melanomas of unusual primary sites; no prior excisions or destruction of skin lesions without a pathologic examination; and no other detectable metastases at diagnosis, after a detailed checkup that included computed tomography imaging (neck, chest, and abdomen).

Radical LNDs were performed between January 1992 and November 2010.

For the final examination, 103 formalin-fixed, paraffinembedded tumor tissues from MUP lymph node metastases were selected, after pathological confirmation (40 from CCIO, 7 from IGR, 52 from Erasmus MC, and 4 from MU). There was access to complete clinical data including date of LDN, date of disease relapse, last follow-up, or death for all patients.

Patient characteristics are summarized in Table 1. The study was approved by the local Bio-Ethics Committee according to Good Clinical Practice Guidelines.

The patients had not undergone any other preliminary selection. Only patients who met with all the conditions listed previously were enrolled in the study. All patients were followed carefully with a median follow-up time of 53 months for survivors (range 6-140 months) with 
TABLE 1 Patient characteristics and comparison between $B R A F$-mutant and $B R A F$ wild-type melanomas in stage III melanomas with unknown primary site

\begin{tabular}{|c|c|c|c|c|}
\hline & $N(\%) 103(100 \%)$ & $\begin{array}{l}B R A F \text { mutants } \\
N(\%) 55\end{array}$ & $\begin{array}{l}B R A F \text { wild type } \\
N(\%) 48\end{array}$ & $p$ value $B R A F+$ versus - \\
\hline Age, median (years) & 54.5 & 51.5 & 56.5 & ns $(p=.08)$ \\
\hline Age groups (years) & & & & ns \\
\hline $0-40$ & $21(17.6)$ & $13(23.7)$ & $9(18.8)$ & \\
\hline$>40-60$ & $45(47.2)$ & $27(49.0)$ & $18(37.5)$ & \\
\hline 60 & $36(35.2)$ & $15(27.3)$ & $21(43.7)$ & \\
\hline Gender & & & & ns \\
\hline Female & $47(45.6)$ & $25(45.5)$ & $22(45.8)$ & \\
\hline Male & $56(54.4)$ & $30(54.5)$ & $26(54.2)$ & \\
\hline Center & & & & ns \\
\hline CCIO Warsaw & $40(39)$ & $25(45)$ & $15(31)$ & \\
\hline Erasmus MS, Rotterdam & $52(50)$ & $25(45)$ & $27(56.5)$ & \\
\hline IGR, Paris & $7(7)$ & $3(6)$ & $4(8.5)$ & \\
\hline MU, Lodz & $4(4)$ & $2(4)$ & $2(4)$ & \\
\hline Lymph nodal basin & & & & ns \\
\hline Axillary & $40(39)$ & $25(45)$ & $15(31)$ & \\
\hline Inguinal & $47(46)$ & $23(42)$ & $24(50)$ & \\
\hline Cervical & $16(15)$ & $7(13)$ & $9(19)$ & \\
\hline Number of metastatic nodes & & & & ns \\
\hline 1 & $37(36)$ & $18(32)$ & $19(40)$ & \\
\hline $2-3$ & $20(19)$ & $12(21)$ & $8(17)$ & \\
\hline$\geq 4$ & $46(45)$ & 19 (34) & 27 (58) & \\
\hline Median & 3 & 3 & 3 & \\
\hline $\begin{array}{l}\text { Extracapsular extension of metastatic node } \\
\text { (data not available in } 40 \text { cases) }\end{array}$ & & & & ns \\
\hline Present & 27 (43) & $15(38)$ & $12(50)$ & \\
\hline Absent & $36(57)$ & $24(62)$ & $12(50)$ & \\
\hline
\end{tabular}

ns not significant statistically

standard postoperative follow-up protocol (surveillance recommended every 3 months for the first 2 years, every 4 months in year 3, every 6 months for years $4-5$, and annually thereafter). Patients were not treated with any BRAF or MEK inhibitors.

\section{Mutational Testing}

A total of 103 paraffin blocks from lymph nodes metastases were selected ( 1 per patient), with the sufficient tumor load and best possible material quality, as described previously. ${ }^{23}$ Samples were cut from the whole block surface. Genomic DNA was isolated with the Sherlock AX DNA kit (A\&A Biotechnology, Gdynia, Poland) and amplified in standard polymerase chain reaction conditions with in-house designed primers for BRAF exons 11, 15, NRAS exons 1, 2, and KIT exons 9, 11, 13, 17. Products were bidirectly sequenced with the BigDye Terminator
Cycle Sequencing Kit and ABI Prism 3100 Genetic Analyzer (Applied Biosystems, Carlsbad, CA, USA). In order to identify mutations, the sequences were aligned to the BRAF (NM_004333.4), NRAS (NM_002524.4), and KIT (NM_000222.2) GenBank references.

\section{Statistical Analyses}

All statistical analyses were performed using R 2.15.1 statistical software (R Core Team (2012); http://www.Rproject.org). Contingency tables were analyzed using the Chi square test. The nonparametric Mann-Whitney $U$ test was applied for comparisons of 2 groups with non-normal distribution.

For survival analyses, the Kaplan-Meier estimator was used with log-rank tests for bivariate comparisons. OS time for the assessment of prognostic value of clinical, pathological, and molecular parameters was calculated from the 

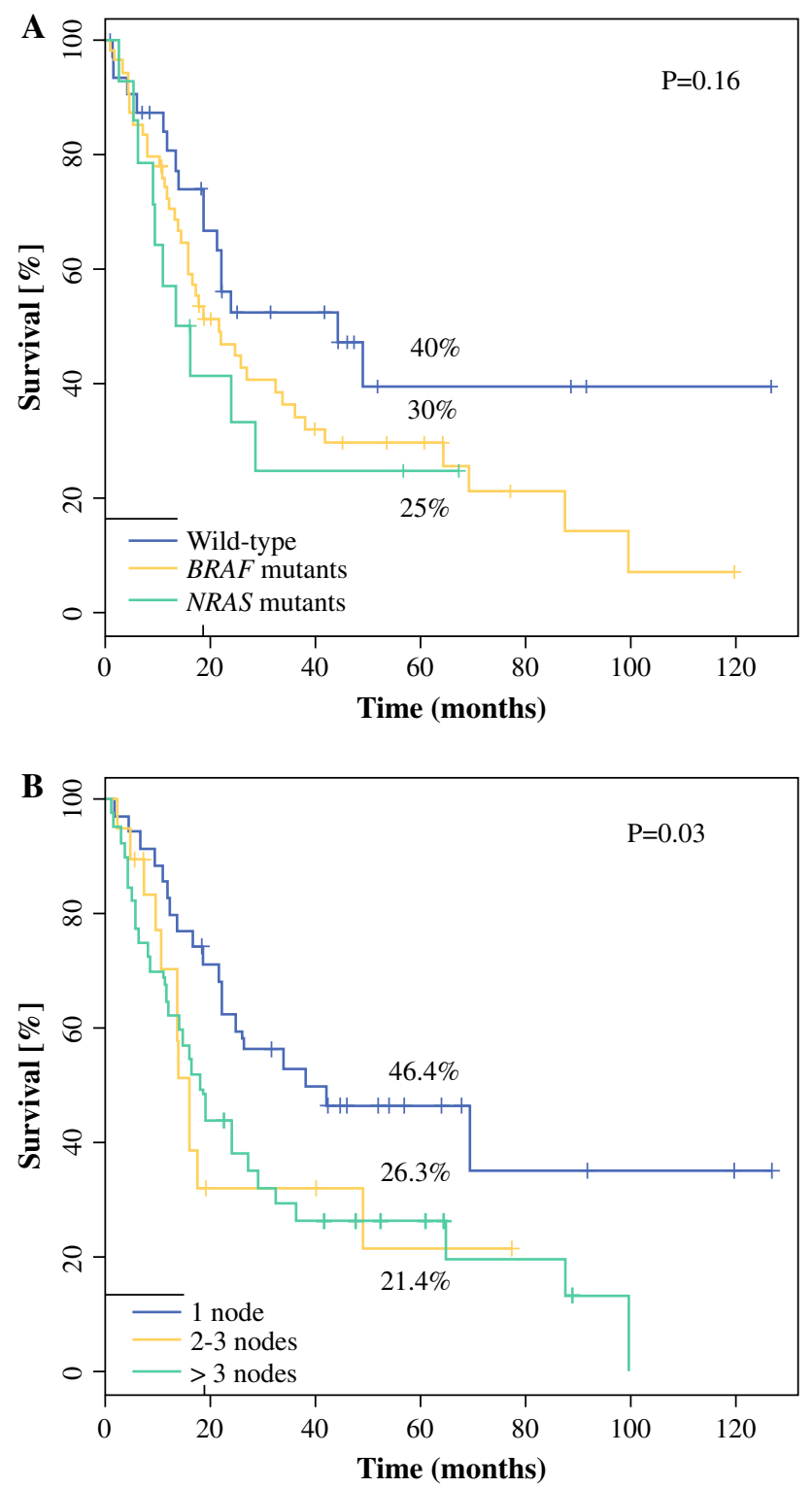

FIG. 1 Overall survival according to: a $B R A F$ and $N R A S$ mutational status in melanomas with unknown primary site and nodal involvement and $\mathbf{b}$ to number of nodes with metastases

date of LND to the date of the most recent follow-up (censored data) or death. DFS time was calculated from the date of therapeutic lymphadenectomy to the date of the most recent follow-up or disease recurrence.

The following clinical, pathological, and molecular parameters were tested as potential factors affecting patient survival: gender, age ( $\leq 40$ vs $40-60$ vs $>60$ years), LND localization (groin vs axillary vs cervical), number of lymph nodes with metastases ( 1 vs $2-3$ vs $\geq 4$ ), presence of extracapsular invasion in the involved lymph nodes, $B R A F$ status (BRAF mutated vs wild type), and NRAS status (NRAS mutated vs wild type).
The differences were considered statistically significant if the respective $p$ values were $<.05$.

\section{RESULTS}

Mutational Status and Correlation with Clinicopathological Features

$B R A F$ mutations were detected in 55 of 103 cases $(53.4 \%)$ of melanoma nodal metastases. Majority of mutations (54 of 55) affected codon 600: 51 were p.V600E $(92.7 \%), 2$ were p.V600 K (3.6\%), and 1 was p.V600_K601delinsE codon deletion $(1.8 \%)$. The only mutation outside codon 600 was p.E586K. A total of 48 samples were wild type for $B R A F$ exons 11 and 15 .

The analysis of NRAS gene status revealed 14 mutated cases, 13 mutations affected codon 61 (7 p.Q61R, 4 p.Q61K, 2 p.Q61H) and 1 affected codon 13 (p.Q13R). NRAS gene was mutated in $13.7 \%$ of all samples, $29 \%$ of $B R A F-W T$ samples. Mutations in $B R A F$ and NRAS were mutually exclusive, and 34 samples harbored neither. No KIT mutations were detected in analyzed cases.

All cases with weak ( $<30 \%$ of BRAF/NRAS wild-type) mutation sequence peak were resequenced, resulting in complete confirmation of the results.

The presence of BRAF mutation had trended to correlate only with younger age of patients, with borderline significance (median, 51.5 vs 56.5 years for $B R A F+$ vs $B R A F-$; $p=.08$ ) (Table 1). We found no differences in patient characteristics when analyzed according to NRAS mutational status. There were no differences in terms of mutation distribution between participating centers.

\section{Treatment Outcomes}

The 5-year OS and DFS rates were $34 \%$ [95\% confidence interval (95\% CI) 25-46\%] and $24 \%$ (95\% CI 16-35\%), respectively; median OS and DFS were 24 months (95\% CI 18.2-36.2 months) and 9 months (95\% CI 6-12 months). There were no significant differences in OS and DFS between patients with $B R A F$ - or $N R A S$-mutated melanoma and those with no mutation. The 5-year survival rates were 36.0 and $29.8 \%$ for patients with wild-type and mutated $B R A F$ genotype $(p=.27)$, respectively; 33.9 and $26.0 \%$ for patients with wild-type and mutated NRAS genotype $(p=.31)$, respectively. Median OS for patients with BRAF mutation versus NRAS mutation versus wild-type were: 21.9 versus 15.1 versus 43.3 months, respectively. The trend for patients with acknowledged mutations to have worse OS has not reached statistical significance $(p=.16)$ (Fig. 1a). 


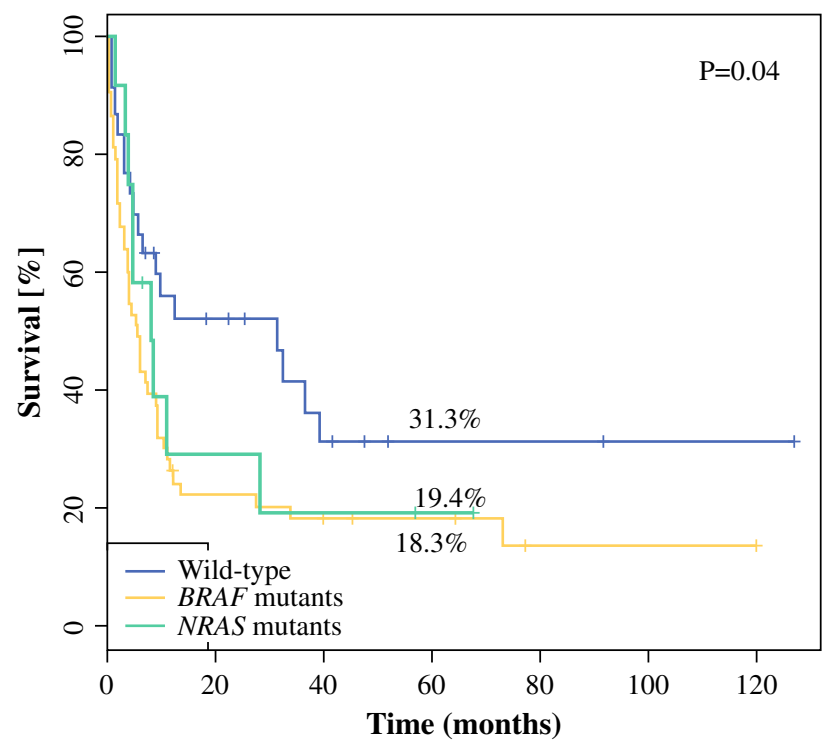

FIG. 2 Disease-free survival (DFS) according to $B R A F$ and $N R A S$ mutational status in melanomas with unknown primary site and nodal involvement

The only negative factor that influenced OS significantly was the number of lymph nodes involved $>1$ (5-year OS rates, 46.4 vs 21.4 vs $26.3 \%$ for 1 vs $2-3$ vs $>3$ metastatic lymph nodes, respectively; $p=.03$ ) (Fig. 1b). There was also a trend for poorer survival in patients with extracapsular extension of nodal metastases, but it did not reach statistical significance (5-year OS rates, 47 vs $33.5 \%$ for absence and presence of extracapsular involvement, respectively; $p=.1$ ).

The 5-year DFS rates were 28.5 and $18.3 \%$ for patients without and with $B R A F$ mutation, respectively (median DFS, 11.8 vs 5.6 months; $p=.03$ ), and 31.3, 18.3 , and $19.4 \%$ for patients without any mutation and with gene alternations in BRAF and NRAS, respectively (median, 31.5 vs 5.6 vs 8.3 months; $p=.04$ ) (Fig. 2). Presence of BRAF/NRAS mutations was related to significantly shorter DFS.

\section{DISCUSSION}

In this study, we analyzed the large and clinically homogeneous group of lymph node metastases that were the first clinical manifestation of advanced melanoma without known primary origin. We have performed the largest mutational analyses in MUP metastatic nodes specimens and more comprehensive survival evaluation in respect to BRAF/NRAS status than has been published to date. $^{23,24}$
Mutation distribution in the MUP study group was similar to those observed in other groups with melanoma of known primary site at stage III and IV and included $53 \%$ of BRAF mutants (with the p.V600E as the most prevalent mutation) and $14 \%$ of mutually exclusive NRAS mutants. ${ }^{6,16,18,25-27}$ No KIT mutations were found.

Based on our mutational data we have shown that a population of MUP patients have a similar distribution of BRAF/NRAS alterations to the known primary melanoma patients exposed to similar/identical environmental factors (such as UV exposure, but not exclusively), confirming data from other smaller published series. ${ }^{16,18,23,24,28}$ The incidence of mutations is consistent with those already observed for melanoma originating on skin without chronic sun damage, which is the most common type in Central and Western Europe. ${ }^{29}$ It implies common pathogenesis for tumor growth of MUP and other skin melanoma types.

There is no unambiguous theory on MUP development mechanism. Widely acceptable is the assumption of spontaneous, immune-induced, complete regression of the primary lesion preceding clinical metastases. ${ }^{30}$ In fact, melanoma is the most common tumor to undergo partial, severe regression, and such events are well documented in primary cutaneous melanoma with frequency of 3-8 \%. ${ }^{31}$ Based on our knowledge of melanoma biology and clinical course, other explanations of MUP origins are also possible and include manifestation as a synchronous, unrecognized melanoma in multiple lesions or malignant transformation of benign nevus cells de novo in a lymph nodes.

The outcomes of our study are especially interesting when compared with reports conducted on different MUP patient populations, where other types of melanoma are dominant. Kong et al. $^{32}$ reported KIT alternations in $13.7 \%$ (including $7.8 \%$ KIT mutations) in a large group of Chinese MUP patients. This is interesting, since acrallentiginous type of melanoma with higher frequency of KIT alternations is the more frequent in Asian patients. However, it could also allude to a proportion of unrecognized mucosal melanomas.

Patients with melanoma of primary unknown site tend to have a prognosis and natural history of disease that is similar to, if not more favorable than, patients with the same staging characteristics, from a known primary cutaneous melanoma. ${ }^{5-7,33-36}$ In our group of MUP patients, survival rates are comparable to previously reported series (5-year OS rates ranged between 28.6 and $75.6 \%$ ) as well as series of stage III patients with macrometastases used for validation of AJCC staging system. ${ }^{7,33}$ It seems that our multicenter group of MUP patients with nodal involvement have outcomes not better than patients with detectable primary tumor according to AJCC database. It implies that they may derive from undetectable primary lesions in the past that gave nodal metastases with lead time bias. 
However, the results should be interpreted with caution, because alternative explanation of de novo development of the tumor within lymph node experiencing a different microenvironment from the start, resulting in aggressive tumor behavior, is also possible.

There is no agreement on prognostic features in MUP with nodal metastases; however, most commonly accepted are female gender, younger age at diagnosis, and smaller number of lymph nodes involvement. ${ }^{4-7}$ We have demonstrated that MUP stage III patients have the same important prognostic factor as known primary melanomas with clinically detected lymph node metastases (stage IIIB, C), e.g., nodal metastases burden expressed as number of metastatic nodes. ${ }^{35}$

The nodal metastases in MUP patients harboring BRAF mutation has a tendency to occur in younger age, which is consistent with observation that p.V600E $B R A F$-mutated melanomas more frequently affect younger individuals with lower cumulative UV exposure..$^{25,37,38}$ OS was not dependent on mutational status (although a trend for poorer prognosis in BRAF/NRAS mutated cases was visible without reaching statistical significance, which may be related to insufficient number of patients); this has been seen previously in advanced melanomas. ${ }^{11,18}$ This study did demonstrate that the presence of BRAF and NRAS mutations had negative impact on DFS in this group of patients. ${ }^{19,39}$

Because the manifestation of melanoma as MUP does not alter $B R A F$ or NRAS mutation frequency compared with melanomas with known primary site, some other genetic/immunological mechanism enhancing regression must be involved. ${ }^{28}$ Based on previously published data and our clinical data on MUP patients, supported by mutational status of known genes involved in development of melanoma as well as potential targets of new therapies, we conclude that MUP has similar molecular pattern in terms of $B R A F / N R A S$ alterations and clinical behavior to melanoma of known primary site of the same stage (mainly developed in NCSD) and therefore should be managed and treated according to the same guidelines. It has important implications for potential adjuvant therapy as well as treatment of metastatic disease in an era of personalized medicine and strongly suggests that MUP with nodal involvement should be included for clinical trials with targeted therapy at stage III disease. BRAF and NRAS gene mutational status does not affect patient outcome significantly, although patients harboring mutations in those genes have less favorable DFS.

ACKNOWLEDGMENT We thank Daniel Rabczenko for statistical advice. The study was supported by the Polish National Science Centre Grant No. 2011/03/B/NZ5/04513.
CONFLICT OF INTEREST The authors declare that they have no conflict of interest.

OPEN ACCESS This article is distributed under the terms of the Creative Commons Attribution License which permits any use, distribution, and reproduction in any medium, provided the original author(s) and the source are credited.

\section{REFERENCES}

1. Morton DL, Wanek L, Nizze JA, Elashoff RM, Wong JH. Improved long-term survival after lymphadenectomy of melanoma metastatic to regional nodes. Analysis of prognostic factors in 1134 patients from the John Wayne Cancer Clinic. Ann Surg. 1991;214:491-501.

2. Katz KA, Jonasch E, Hodi FS, Soiffer R, Kwitkiwski K, Sober AJ, et al. Melanoma of unknown primary: Experience at Massachusetts General Hospital and Dana-Farber Cancer Institute. Melanoma Res. 2005;15:77-82.

3. Jonk A, Kroon BB, Rumke P, Mooi WJ, Hart AA, van Dongen JA. Lymph node metastasis from melanoma with an unknown primary site. Br J Surg. 1990;77:665-8.

4. Cormier JN, Xing Y, Feng L, Huang X, Davidson L, Gershenwald JE, et al. Metastatic melanoma to lymph nodes in patients with unknown primary sites. Cancer. 2006;106:2012-20.

5. Rutkowski P, Nowecki ZI, Dziewirski W, Zdzienicki M, Pieñkowski A, Salamacha M, et al. Melanoma without a detectable primary site with metastases to lymph nodes. Dermatol Surg. 2010;36:868-76.

6. Lee CC, Faries MB, Wanek LA, Morton DL. Improved survival after lymphadenectomy for nodal metastasis from an unknown primary melanoma. J Clin Oncol. 2008;26:535-41.

7. Kamposioras K, Pentheroudakis G, Pectasides D, Pavlidis N. Malignant melanoma of unknown primary site. To make the long story short. A systematic review of the literature. Crit Rev Oncol Hematol. 2011;78:112-26.

8. Balch CM, Gershenwald JE, Soong SJ, Thompson JF, Atkins MB, Byrd DR, et al. Final version of 2009 AJCC melanoma staging and classification. J Clin Oncol. 2009;27:6199-206.

9. Curtin JA, Busam K, Pinkel D, Bastian BC. Somatic activation of KIT in distinct subtypes of melanoma. $J$ Clin Oncol. 2006;24:4340-6.

10. Kyrgidis A, Tzellos TG, Triaridis S. Melanoma: stem cells, sun exposure and hallmarks for carcinogenesis, molecular concepts and future clinical implications. J Carcinog. 2010;9:3.

11. Ellerhorst JA, Greene VR, Ekmekcioglu S, Warneke CL, Johnson $\mathrm{MM}$, Cooke CP, et al. Clinical correlates of NRAS and BRAF mutations in primary human melanoma. Clin Cancer Res. 2011;17:229-35.

12. Ascierto PA, Berking C, Agarwala SS Schadendorf D, Van Herpen C, Queirolo P, et al. Efficacy and safety of oral MEK162 in patients with locally advanced and unresectable or metastatic cutaneous melanoma harboring BRAFV600 or NRAS mutations. J Clin Oncol. 2012;30(Suppl);Abstr 8511.

13. Davies H, Bignell GR, Cox C, Stephens P, Edkins S, Clegg S, et al. Mutations of the BRAF gene in human cancer. Nature. 2002;417:949-54.

14. Wellbrock C, Hurlstone A. BRAF as therapeutic target in melanoma. Biochem Pharmacol. 2010;80:561-7. 
15. Pollock PM, Harper UL, Hansen KS, Yudt LM, Stark M, Robbins $\mathrm{CM}$, et al. High frequency of BRAF mutations in nevi. Nat Genet. 2003;33:19-20.

16. Colombino M, Capone M, Lissia A, Cossu A, Rubino C, De Giorgi $\mathrm{V}$, et al. BRAF/NRAS mutation frequencies among primary tumors and metastases in patients with melanoma. J Clin Oncol. 2012;30:2522-9.

17. Jakob JA, Bassett RL Jr, Ng CS, Curry JL, Joseph RW, Alvarado $\mathrm{GC}$, et al. NRAS mutation status is an independent prognostic factor in metastatic melanoma. Cancer. 2012;118:4014-23.

18. Long GV, Menzies AM, Nagrial AM, Haydu LE, Hamilton AL, Mann GJ, et al. Prognostic and clinicopathologic associations of oncogenic BRAF in metastatic melanoma. $J$ Clin Oncol. 2011;29:1239-46.

19. Moreau S, Saiag P, Aegerter P, Bosset D, Longvert C, HéliasRodzewicz Z, et al. Prognostic value of BRAFV600 mutations in melanoma patients after resection of metastatic lymph nodes. Ann Surg Oncol. 2012;19:4314-21.

20. Chapman PB, Hauschild A, Robert C, Haanen JB, Ascierto P, Larkin $\mathrm{J}$, et al. Improved survival with vemurafenib in melanoma with BRAF V600E mutation. $N$ Engl J Med. 2011;364:2507-16.

21. Hauschild A, Grob JJ, Demidov LV, Jouary T, Gutzmer R, Millward M, et al. Dabrafenib in BRAF-mutated metastatic melanoma: a multicentre, open-label, phase 3 randomised controlled trial. Lancet. 2012;380:358-65.

22. Ascierto PA, Kirkwood JM, Grob JJ, Simeone E, Grimaldi AM, Maio M, et al. The role of BRAF V600 mutation in melanoma. $J$ Transl Med. 2012;10:85.

23. Dutton-Regester K, Kakavand H, Aoude LG, Stark MS, Gartside MG, Johansson P, et al. Melanomas of unknown primary have a mutation profile consistent with cutaneous sun-exposed melanoma. Pigment Cell Melanoma Res. 2013;26:852-60.

24. Egberts F, Bergner I, Kruger S, Haag J, Behrens HM, Hauschild A, et al. Metastatic melanoma of unknown primary resembles the genotype of cutaneous melanomas. Ann Oncol. 2014;25:246-50.

25. Rutkowski P, Gos A, Jurkowska M, Switaj T, Dziewirski W, Zdzienicki $\mathrm{M}$, et al. Molecular alterations in clinical stage III cutaneous melanoma: correlation with clinicopathological features and patient outcome. Oncol Lett. 2014;8:47-54.

26. Edlundh-Rose E, Egyházi S, Omholt K, Månsson-Brahme E, Platz A, Hansson J, et al. NRAS and BRAF mutations in melanoma tumours in relation to clinical characteristics: a study based on mutation screening by pyrosequencing. Melanoma Res. 2006; 16:471-8.

27. Greaves WO, Verma S, Patel KP, Davies MA, Barkoh BA, Galbincea JM, et al. Frequency and spectrum of BRAF mutations in a retrospective, single-institution study of 1112 cases of melanoma. J Mol Diagn. 2013;15:220-6.

28. Egberts F, Kruger S, Behrens HM, Bergner I, Papaspyrou G, Werner JA, et al. Melanomas of unknown primary frequently harbor TERT-promoter mutations. Melanoma Res. 2014;24: $131-6$.

29. Curtin JA, Fridlyand J, Kageshita T, Patel HN, Busam KJ, Kutzner $\mathrm{H}$, et al. Distinct sets of genetic alterations in melanoma. $N$ Engl J Med. 2005;353:2135-47.

30. Anbari KK, Schuchter LM, Bucky LP, Mick R, Synnestvedt M, Guerry D 4th, et al. Melanoma of unknown primary site: presentation, treatment, and prognosis-a single institution study. University of Pennsylvania Pigmented Lesion Study Group. Cancer. 1997;79:1816-21.

31. Blessing K, McLaren KM, Histological regression in primary cutaneous melanoma: recognition, prevalence and significance. Histopathology. 1992;20:315-22.

32. Kong Y, Si L, Zhu Y, Xu X, Corless CL, Flaherty KT, et al. Large-scale analysis of KIT aberrations in Chinese patients with melanoma. Clin Cancer Res. 2011;17:1684-92.

33. Prens SP, van der Ploeg AP, van Akkooi AC, van Montfort CA, van Geel AN, de Wilt JH, et al. Outcome after therapeutic lymph node dissection in patients with unknown primary melanoma site. Ann Surg Oncol. 2011;18:3586-92.

34. de Waal AC, Aben KK, van Rossum MM, Kiemeney LA. Melanoma of unknown primary origin: a population-based study in the Netherlands. Eur J Cancer. 2013;49:676-83.

35. Balch CM, Gershenwald JE, Soong SJ, Thompson JF, Ding S, Byrd DR, et al. Multivariate analysis of prognostic factors among 2,313 patients with stage III melanoma: comparison of nodal micrometastases versus macrometastases. J Clin Oncol. 2010;28: 2452-9.

36. Pfeil AF, Leiter U, Buettner PG, Eigentler TK, Weide B, Meier F, et al. Melanoma of unknown primary is correctly classified by the AJCC melanoma classification from 2009. Melanoma Res. 2011;21:228-34.

37. Liu W, Kelly JW, Trivett M, Murray WK, Dowling JP, Wolfe R, et al. Distinct clinical and pathological features are associated with the BRAF(T1799A(V600E)) mutation in primary melanoma. J Invest Dermatol. 2007;127:900-5.

38. Menzies AM, Haydu LE, Visintin L, Carlino MS, Howle JR, Thompson JF, et al. Distinguishing clinicopathologic features of patients with V600E and V600 K BRAF-mutant metastatic melanoma. Clin Cancer Res. 2012;18:3242-9.

39. Houben R, Becker JC, Kappel A, Terheyden P, Bröcker EB, Goetz R, et al. Constitutive activation of the Ras-Raf signaling pathway in metastatic melanoma is associated with poor prognosis. J Carcinog. 2004;3:6. 